\title{
Hyperlipidemia and hypertension have synergistic interaction on ischemic stroke: insights from a general population survey in China
}

\author{
Chang Wang ${ }^{1}$, Zhi Du ${ }^{2}$ Ning Ye ${ }^{1}$, Chuning Shi', Songyue Liu' ${ }^{1}$, Danxi Geng ${ }^{1}$ and Yingxian Sun ${ }^{1 *}$
}

\begin{abstract}
Background: Hyperlipidemia (HLP) and hypertension (HTN) are both independent risk factors for ischemic stroke. This study aimed to assess whether HTN and HLP have a synergistic effect on the risk of ischemic stroke.

Methods: Between January and August 2013, 11,695 subjects in rural areas of northeastern China were enrolled. The additive and multiplicative scales were used to evaluate the interaction.

Results: The prevalence of ischemic stroke was 5.7\%. Using the healthy group (without HTN or HLP) as the reference group, subjects with both HTN and HLP had a higher risk of ischemic stroke (odds ratio [OR]: 3.369, 95\% confidence interval [Cl]: 2.579-4.402), and this OR was greater than that of subjects with only HTN (OR: 1.995, 95\% Cl 1.526-2.610) or HLP (OR: 1.321, 95\% Cl 0.937-1.862) (adjusting for age, sex, race, education level, family income, current smoking and drinking status, physical activity, body mass index, diabetes, family history of stroke, and atrial fibrillation). Regarding the additive scale, the relative excess risk due to interaction (OR: 1.053, 95\% Cl 0.458-1.648) was positive after adjusting for confounders. Moreover, the attributable proportion was $31.3 \%$, which means that $31.3 \%$ of the total risk of ischemic stroke was due to the synergistic interaction between HTN and HLP. Furthermore, the synergistic index (S) of ischemic stroke was $1.8(95 \% \mathrm{Cl} 1.157-2.801)$, which also indicates a synergistic interaction between HTN and HLP. Regarding the multiplicative scale, the interaction effect was also significant after adjusting for confounders (OR: $2.163,95 \%$ Cl 1.817-2.575).
\end{abstract}

Conclusion: The results suggest that the synergistic effect of HTN and HLP on ischemic stroke is significantly higher than the sum of their independent effects. The quantification of the combined effect should help to promote healthy blood pressure and blood lipid levels among the general population.

Keywords: Hypertension, Hyperlipidemia, Ischemic stroke, Synergistic interaction, Epidemiology

\footnotetext{
*Correspondence: sunyingxian_cmu@126.com

1 Department of Cardiovascular Medicine, The First Hospital of China

Medical University, ShenyangLiaoning 110001, China

Full list of author information is available at the end of the article
}

\section{Background}

Stroke has become a primary cause of disability and death in recent years, which has greatly increased the global healthcare burden [1]. In China, the incidence of stroke is about 157 per 100,000 people, exceeding that of heart disease, and it continues to increase annually [2]. Notably, the proportion of ischemic stroke among stroke cases is extremely high among low-income groups in 
rural China [3], underscoring the importance to screen for individuals with a high risk of ischemic stroke and providing preventive measures. To improve these interventions, the risk factors for stroke and their interactions should be explored in depth.

Hypertension (HTN) is an independent risk factor for ischemic stroke. Over $60 \%$ of acute stroke patients have elevated blood pressure [4]. Large-scale cohort studies have also shown that effective blood pressure control can reduce the occurrence of ischemic stroke [5-7]. Hyperlipidemia (HLP) is another very dangerous risk factor that can lead to cardiovascular and cerebrovascular diseases, especially atherosclerosis [8]. Moreover, HLP is an independent risk factor for ischemic stroke [9]. Statin use to control blood lipids plays an important role in primary and secondary stroke prevention $[10,11]$, as statins can promote neurogenesis in many ways, including by inhibiting platelet aggregation and clot formation, regulating inflammation, and inhibiting nitric oxide metabolism and endothelial nitric oxide synthase $[8,12]$. Both HTN and HLP contribute to thickening of the carotid artery intima-media, thereby reducing the blood supply to the brain and leading to ischemic stroke [13, 14].

Potential associations among HTN, HLP and ischemic stroke have been reported in previous studies $[15,16]$. However, they only investigated the independent effects of risk factors and did not consider interactions. To date, no studies have assessed the combined effect of HTN plus HLP on ischemic stroke. It is very common for patients to have both risk factors. Therefore, we aimed to assess whether there is a significant interaction between HTN and HLP regarding ischemic stroke, leading to a greater combined effect than the sum of their independent effects. This study also sought to quantify the synergistic interaction and provide an intuitive description for the public to increase awareness of the risk and promote healthy blood pressure and blood lipid levels.

\section{Materials and methods Study population}

The study employed data from the Northeast China Rural Cardiovascular Health Study (NCRCHS), which was a cross-sectional epidemiological survey implemented from January to August 2013. A multistage, stratified, random cluster sampling was used. The full details of the design and principles of the study were described elsewhere [17-19].

After excluding pregnant subjects and those with cancer or mental disorders, 11,956 residents aged $\geq 35$ years completed the baseline survey. After excluding a further 261 subjects due to missing data, 11,695 subjects were included in the study.
The Ethics Committee of China Medical University (Shenyang, China) approved the research protocol. Subjects who had undergone preliminary screening and examination were included after signing a written informed consent form, and all data collection and other procedures complied with ethical standards.

\section{Data collection and measurements}

Our previous studies described the detailed data collection process [20-22]. The cardiologists and nurses who collected the data completed professional training before starting the study.

For each subject, trained medical staff members administered a questionnaire, which included information about anthropometric parameters, dietary intake, family history of diabetes, education level, annual family income, and medication use in the past 2 weeks. Next, two trained medical staff members measured each subject's blood pressure three times (to calculate the mean) using an automated blood pressure monitor (HEM-907; Omron, Tokyo, Japan). This was done with their arm flush with the heart after they had waited in a relaxed sitting position for at least $5 \mathrm{~min}$. Then the anthropometric parameters of each subject (wearing light clothes) were measured twice (to calculate the mean). Weight was measured using a calibrated electronic weight scale (accurate to $0.1 \mathrm{~kg}$ ), height (when standing barefoot) using a portable rangefinder (accurate to $0.1 \mathrm{~cm}$ ), and waist circumference (at the level of the umbilicus) using a non-elastic tape measure (accurate to $0.1 \mathrm{~cm}$ ).

Lastly, fasting (12 h overnight) blood samples were collected via venipuncture into ethylenediaminetetraacetic acid-containing tubes. Within an hour, the plasma was separated and frozen at $-20{ }^{\circ} \mathrm{C}$, and it was then immediately sent for laboratory testing. The biochemical analysis was carried out using an AU640 automatic analyzer (Olympus, Kobe, Japan). Fasting blood glucose, total cholesterol (TC), and low-density lipoprotein cholesterol (LDL-C) were automatically analyzed, along with high-density lipoprotein cholesterol (HDL-C), triglycerides (TG), uric acid, serum creatinine, and other routine blood biochemical parameters. All laboratory equipment was calibrated, and the samples were repeatedly analyzed in a blinded fashion.

\section{Definitions}

Ischemic stroke was diagnosed by neurologists based on World Health Organization standards using computed tomography and magnetic resonance imaging scans. HTN was defined as mean blood pressure $>140 / 90 \mathrm{mmHg}$ or taking HTN medication in the 2 weeks. HLP was defined according to current lipid levels or use of anti-dyslipidemia medication in the 
past two weeks. The cut-off values for hypercholesterolemia, hypertriglyceridemia, and low HDL-cholesterolemia were $\mathrm{TC} \geq 6.22 \mathrm{mmol} / \mathrm{L}(\geq 240 \mathrm{mg} / \mathrm{dL})$, $\mathrm{TG} \geq 2.26 \mathrm{mmol} / \mathrm{L}(\geq 200 \mathrm{mg} / \mathrm{dL})$, and HDL-cholesterol $\leq 1.04 \mathrm{mmol} / \mathrm{L}(40 \mathrm{mg} / \mathrm{dL})$, respectively [23]. Diabetes was defined as fasting blood glucose $>7.0 \mathrm{mmol} / \mathrm{L}$, self-reported diabetes, or currently taking hypoglycemic medication. Body mass index (BMI) was defined as weight divided by the square of height.

\section{Statistical analysis}

Continuous variables are presented as mean \pm standard deviation, and categorical variables are presented as frequency (percentage). Student's $\mathrm{t}$-tests or the chi-squares test were used to compare the ischemic and non-ischemic stroke groups. Multivariate logistic regression was used to assess the associations of HLP and HTN with ischemic stroke, expressed as odds ratios (ORs) with 95\% confidence intervals (CIs). Many variables were adjusted, including age, sex, race, education level, family annual income, current smoking and drinking status, BMI, TC, HDL-C, and diabetes (see table footnotes for the specific variables adjusted in each analysis).

To clarify the association of HLP combined with HTN with ischemic stroke, we analyzed the synergistic interaction using both the additive and multiplicative scales $[24,25]$. If the risk of two coexisting risk factors is significantly greater than the sum of their independent risks, there is an additive interaction. If the risk of two coexisting risk factors is significantly greater than the product of their independent risks, there is a multiplicative interaction.

To calculate the additive interaction, we determined the relative excess risk due to interaction (RERI; which represents the relative excess OR value caused by the interaction), along with the attributable proportion due to interaction (AP) and the synergy index (S) $[26,27]$. RERI $=(\mathrm{ORHTN}+\mathrm{HLP}-\mathrm{O}$ RHTN-ORHLP) +1 , where ORHTN + HLP is the OR of ischemic stroke when HTN and HLP coexist, ORHTN is the OR of ischemic stroke when the subject has only HTN, and ORHLP is the OR of ischemic stroke when the subject has only HLP. AP = RERI/ ORHTN + HLP and S index $=[$ ORHTN + HLP -1$] /$ $[($ ORHTN -1$)+($ ORHLP -1$)]$. RERI and AP are significant if the $95 \%$ CI does not cross 0 , and the $S$ index is significant if the $95 \% \mathrm{CI}$ does not cross 1 . To calculate the multiplicative interaction, HTN $\times$ HLP was included as an independent factor in the regression analysis, and its OR and $P$ values were used to evaluate the magnitude and significance of the multiplicative interaction.
The analyses were performed in SPSS 25.0 software (IBM Corp., Armonk, NY, USA) and Prism 8.0 software (GraphPad Software, Inc, San Diego, CA, USA).

\section{Results}

The study involved data from 11,695 patients. Table 1 shows the basic characteristics of the ischemic stroke and non-ischemic stroke groups. The number of patients with ischemic stroke was 667 (5.70\%). The prevalence of HTN in the ischemic stroke group (78.3\%) was significantly higher than in the non-ischemic stroke group (48.8\%). Similarly, TC, TG, and LDL-C were higher in the ischemic stroke group than the non-ischemic stroke group, while HDL-C was lower in the ischemic stroke group. The prevalence of HLP in the ischemic stroke group (38.5\%) was significantly higher than in the nonischemic stroke group (37.5\%).

To investigate the relationships of HTN and HLP with ischemic stroke, we conducted logistic regression analysis. The results showed that HTN was significantly associated with ischemic stroke (OR: 2.299, 95\% CI 1.877-2.816) after adjusting for age, sex, race, education level, family annual income, current smoking and drinking status, BMI, TC, HDL-C, and diabetes. HLP was also an independent risk factor for ischemic stroke (OR: 1.655 , 95\% CI 1.397-1.959) after adjusting for the same variables (Table 2).

The subjects were divided into four categories (nonHTN + non-HLP, non-HTN + HLP, HTN + non-HLP, and HTN + HLP) to assess the effects of HTN and HLP on ischemic stroke risk (Table 3). In the unadjusted model, the OR for subjects with both HTN and HLP compared to subjects with neither HTN nor HLP was 5.907 (95\% CI 1.078-2.111, $P<0.001$ ); the OR was also much higher than that for subjects with only HTN (OR: $3.168,95 \%$ CI $2.455-4.089, P<0.001$ ) or HLP (OR: 1.509 , 95\% CI 1.078-2.111, $P<0.001)$. These differences persisted after adjustment; subjects with both HTN and HLP had a higher risk of ischemic stroke (OR: 3.369, 95\% CI 2.579-4.402, $P<0.001)$ compared to subjects with only HTN (OR: $1.995,95 \%$ CI $1.526-2.610, P<0.001$ ) or HLP (OR: $1.321,95 \%$ CI $0.937-1.862, P=0.112$ ). In a subsequent stratified analysis, the risk of ischemic stroke in HTN patients differed by HLP status: the risk was higher in the HLP group than in the non-HLP group. The risk of ischemic stroke in HLP patients was also slightly higher in the HTN group than in the non-HTN group.

To determine whether HTN and HLP have a synergistic effect on ischemic stroke, rather than the effect being the sum of their independent effects, we calculated the interaction effects using additive and multiplicative scales (Table 4). Regarding the additive scale, the RERI (OR: 1.053, 95\% CI 0.458-1.648) was positive after 
Table 1 Characteristics of subjects by ischemic stroke status

\begin{tabular}{|c|c|c|c|}
\hline Variable & $\begin{array}{l}\text { Non-ischemic stroke } \\
(n=11,028)\end{array}$ & $\begin{array}{l}\text { Ischemic stroke } \\
(n=667)\end{array}$ & $p$ value \\
\hline Age $(y)$ & $53.46 \pm 10.50$ & $61.26 \pm 9.42$ & $<0.001$ \\
\hline Males (\%) & $5019(46.3)$ & $306(45.9)$ & 0.821 \\
\hline Ethnicity (Han, \%) & $10,446(94.7)$ & $637(95.5)$ & 0.38 \\
\hline $\mathrm{SBP}(\mathrm{mmHg})$ & $140.98 \pm 22.94$ & $155.27 \pm 27.30$ & $<0.001$ \\
\hline $\mathrm{DBP}(\mathrm{mmHg})$ & $81.79 \pm 11.68$ & $86.37 \pm 12.52$ & $<0.001$ \\
\hline Education status & & & $<0.001$ \\
\hline Primary & $5394(48.9)$ & $430(64.5)$ & \\
\hline Middle & $4566(41.4)$ & $195(29.2)$ & \\
\hline High & $1068(9.7)$ & $42(6.3)$ & \\
\hline Family income (CNY/year, \%) & & & $<0.001$ \\
\hline$\leq 5000$ & $832(7.5)$ & $117(17.5)$ & \\
\hline $5000-20,000$ & $6458(58.6)$ & $419(62.8)$ & \\
\hline$>20,000$ & 3738 (33.9) & $131(19.6)$ & \\
\hline Exercise (\%) & $2315(21)$ & $230(34.5)$ & $<0.001$ \\
\hline Current smoking (\%) & $3896(35.3)$ & $223(33.4)$ & 0.32 \\
\hline Current drinking (\%) & $2540(23)$ & $85(12.7)$ & $<0.001$ \\
\hline $\mathrm{BMI}\left(\mathrm{kg} / \mathrm{m}^{2}\right)$ & $24.78 \pm 3.70$ & $25.24 \pm 3.66$ & $<0.001$ \\
\hline $\mathrm{FBG}(\mathrm{mmol} / \mathrm{L})$ & $5.87 \pm 1.59$ & $6.40 \pm 2.14$ & $<0.001$ \\
\hline $\mathrm{TC}(\mathrm{mmol} / \mathrm{L})$ & $5.22 \pm 1.08$ & $5.47 \pm 1.12$ & $<0.001$ \\
\hline $\mathrm{TG}(\mathrm{mmol} / \mathrm{L})$ & $1.61 \pm 1.45$ & $2.02 \pm 1.95$ & $<0.001$ \\
\hline LDL-C (mmol/L) & $2.92 \pm 0.82$ & $3.11 \pm 0.86$ & $<0.001$ \\
\hline $\mathrm{HDL}-\mathrm{C}(\mathrm{mmol} / \mathrm{L})$ & $1.41 \pm 0.38$ & $1.32 \pm 0.36$ & $<0.001$ \\
\hline AF (\%) & $79(0.7)$ & $16(2.4)$ & $<0.001$ \\
\hline Diabetes (\%) & $441(4.0)$ & $90(13.5)$ & $<0.001$ \\
\hline Hypertension (\%) & $5385(48.8)$ & $522(78.3)$ & $<0.001$ \\
\hline Hyperlipidemia (\%) & $4131(37.5)$ & $369(55.3)$ & $<0.001$ \\
\hline
\end{tabular}

AF, atrial fibrillation; BMI, body mass index; DBP, diastolic blood pressure; FBG, fasting blood glucose; HDL-C, high-density lipoprotein cholesterol; HLP, hyperlipidemia; HTN, hypertension; LDL-C, low-density lipoprotein cholesterol; SBP, systolic blood pressure; TC, total cholesterol; TG, triglycerides

Table 2 Logistic regression models of the effect of HTN or HLP on the risk of ischemic stroke

\begin{tabular}{lllll}
\hline Model & Risk factor & OR & $\mathbf{9 5 \% ~ C l}$ & $\boldsymbol{p}$ value \\
\hline Unadjusted & HTN & 3.712 & $(3.127-4.552)$ & $<0.001$ \\
& HLP & 2.067 & $(1.766-2.420)$ & $<0.001$ \\
Model 1 & HTN & 2.539 & $(2.133-3.153)$ & $<0.001$ \\
& HLP & 1.915 & $(1.631-2.248)$ & $<0.001$ \\
Model 2 & HTN & 2.299 & $(1.877-2.816)$ & $<0.001$ \\
& HLP & 1.655 & $(1.397-1.959)$ & $<0.001$ \\
\hline
\end{tabular}

Model 1: adjusted for age and sex

Model 2: adjusted for age, sex, race, education level, family annual income, current smoking and drinking status, BMI, TC, HDL-C, and diabetes

$\mathrm{Cl}$, confidence interval; $\mathrm{HLP}$, hyperlipidemia; $\mathrm{HTN}$, hypertension; OR, odds ratio

adjustment, which indicates that HTN and HLP had a significant synergistic effect on ischemic stroke. Moreover, AP showed that $31.3 \%$ of the total risk was due to the synergistic interaction between HTN and HLP. In addition, the S index was 1.8 (95\% CI 1.157-2.801), confirming the synergistic interaction between HTN and HLP. Regarding the multiplicative scale, the interaction was also significant after adjustment (OR: 2.163, 95\% CI 1.817-2.575).

To better express these findings, a clear illustration of the results of the additive interaction analyses is presented in Fig. 1. Subjects with both HTN and HLP had a higher OR of ischemic stroke than the other subjects, and $31.3 \%$ of the OR in subjects with both HTN and HLP was attributable to the synergistic interaction effect.

\section{Discussion}

In rural areas of northeastern China, the associations of HTN and HLP with ischemic stroke were positive and independent. This study is the first to demonstrate that the coexistence of HTN and HLP may have a greater combined effect on ischemic stroke than the sum of their individual effects. In this epidemiological study, we calculated the exact synergistic interaction of HTN and HLP 
Table 3 Logistic regression models of the combined effect of HLP and HTN on ischemic stroke

\begin{tabular}{|c|c|c|c|c|c|}
\hline & \multicolumn{2}{|l|}{ Non-HTN } & \multicolumn{2}{|l|}{ HTN } & \multirow{2}{*}{$\begin{array}{l}\text { HTN by HLP status } \\
\text { OR }(95 \% \mathrm{Cl})\end{array}$} \\
\hline & $\begin{array}{l}\text { No. of cases/non- } \\
\text { cases of ischemic } \\
\text { stroke }\end{array}$ & OR $(95 \% \mathrm{Cl})$ & $\begin{array}{l}\text { No. of cases/non- } \\
\text { cases of ischemic } \\
\text { stroke }\end{array}$ & OR $(95 \% \mathrm{Cl})$ & \\
\hline \multicolumn{6}{|l|}{ Unadjusted model } \\
\hline Non-HLP & $86 / 3879$ & 1 & $212 / 3018$ & $\begin{array}{l}3.168(2.455-4.089) \\
p<0.001\end{array}$ & $\begin{array}{l}3.168(2.455-4.089) \\
p<0.001\end{array}$ \\
\hline HLP & $59 / 1764$ & $\begin{array}{l}1.509(1.078-2.111) \\
p=0.016\end{array}$ & $310 / 2367$ & $\begin{array}{l}5.907(1.078-2.111) \\
p<0.001\end{array}$ & $\begin{array}{l}3.916(2.944-5.208) \\
p<0.001\end{array}$ \\
\hline HLP by HTN status & & $\begin{array}{l}1.509(1.078-2.111) \\
p=0.016\end{array}$ & & $\begin{array}{l}1.864(1.553-2.238) \\
p<0.001\end{array}$ & \\
\hline \multicolumn{6}{|l|}{ Model 1} \\
\hline Non-HLP & $86 / 3879$ & 1 & $212 / 3018$ & $\begin{array}{l}2.087(1.604-2.716) \\
p<0.001\end{array}$ & $\begin{array}{l}2.087(1.604-2.716) \\
p<0.001\end{array}$ \\
\hline HLP & $59 / 1764$ & $\begin{array}{l}1.383(0.986-1.940) \\
p=0.06\end{array}$ & $310 / 2367$ & $\begin{array}{l}3.974(3.092-5.108) \\
p<0.001\end{array}$ & $\begin{array}{l}2.900(2.165-3.884) \\
p<0.001\end{array}$ \\
\hline $\begin{array}{l}\text { HLP within strata of } \\
\text { HTN }\end{array}$ & & $\begin{array}{l}1.383(0.986-1.940) \\
p=0.06\end{array}$ & & $\begin{array}{l}1.896(1.575-2.283) \\
p<0.001\end{array}$ & \\
\hline \multicolumn{6}{|l|}{ Model 2} \\
\hline Non-HLP & $86 / 3879$ & 1 & $212 / 3018$ & $\begin{array}{l}1.995(1.526-2.610) \\
p<0.001\end{array}$ & $\begin{array}{l}1.995(1.526-2.610) \\
p<0.001\end{array}$ \\
\hline HLP & $59 / 1764$ & $\begin{array}{l}1.321(0.937-1.862) \\
p=0.112\end{array}$ & $310 / 2367$ & $\begin{array}{l}3.369(2.579-4.402) \\
p<0.001\end{array}$ & $\begin{array}{l}2.605(1.926-3.523) \\
p<0.001\end{array}$ \\
\hline $\begin{array}{l}\text { HLP within strata of } \\
\text { HTN }\end{array}$ & & $\begin{array}{l}1.321(0.937-1.862) \\
p=0.112\end{array}$ & & $\begin{array}{l}1.708(1.406-2.076) \\
p<0.001\end{array}$ & \\
\hline
\end{tabular}

Model 1: adjusted for age and sex

Model 2: adjusted for age, sex, race, education level, family income, current smoking and drinking status, physical activity, BMI, diabetes, family history of stroke, and atrial fibrillation

$\mathrm{Cl}$, confidence interval; HLP, hyperlipidemia; HTN, hypertension; OR, odds ratio

regarding the risk of ischemic stroke using additive and multiplicative scales. The results provide the public with intuitive and straightforward illustrations to increase awareness and understanding of the risk, which may help to promote healthy blood lipid and blood pressure levels.

Interaction analysis was used to explore whether the combination of the two risk factors leads to different risks compared to the sum of the independent risk factors. There are two possible scales regarding the impact of this interaction: additive and multiplicative. Assessing the combined effect using the additive scale involves assessing the difference between the risk of the outcome when both risk factors are present and the sum of the risks when there is only a single risk factor present. Assessing the combined effect using the multiplicative scale involves assessing the difference between the risk of the outcome when both risk factors are present and the product of the risks when only a single risk factor is present [26, 28]. Having only one significant dimension of interaction is common. To date, no research has shown which scale is better. As explained above, the additive scale can directly evaluate the OR caused by the synergy of two risk factors, so the additive scale may have stronger clinical significance [26, 29]. However, the Strengthening the Reporting of Observational Studies in Epidemiology (STROBE) statement specifies that both additive and multiplicative scales should be provided when investigating combined effects [30]. Therefore, we report the results of the interaction of the two scales.

The additive interaction showed that the combination of HTN and HLP had a synergistic impact on ischemic stroke, which was greater than the sum of the effect of each risk factor. Therefore, our study shows that subjects with both HTN and HLP will not only have an increased impact compared to subjects with either HTN or HLP, but there will also be a synergistic effect. It is therefore important to control both blood pressure and blood lipids to prevent ischemic stroke.

Our research is consistent with the results of previous studies that showed that effective control of HTN can effectively reduce ischemic stroke risk [4, 5, 31]. A Japanese study showed that HTN increases the lifetime risk of ischemic stroke [32]. Early antihypertensive treatment in the acute phase can reduce the stroke recurrence rate [33]. HLP is another independent risk factor for ischemic stroke that can also affect the process of atherosclerosis 
Table 4 Interaction analysis of the effects of HLP plus HTN on risk of ischemic stroke

\begin{tabular}{lll}
\hline Model & Effect value & $\mathbf{9 5 \%} \mathbf{C l}$ \\
\hline Unadjusted model & & \\
Additive scale & & \\
$\quad$ RERl & 2.23 & $(1.278-3.182)$ \\
AP & 0.378 & $(0.248-0.507)$ \\
$\quad$ S index & 1.833 & $(1.376-2.442)$ \\
Multiplicative scale & & \\
OR & 3.177 & $(2.711-3.724)$ \\
Model 1 & & \\
Additive scale & & \\
$\quad$ RERl & 1.504 & $(0.836-2.172)$ \\
AP & 0.378 & $(0.231-0.526)$ \\
S index & 2.023 & $(1.337-3.061)$ \\
Multiplicative scale & & $(2.156-2.984)$ \\
OR & 2.536 & \\
Model 2 & & $(0.458-1.648)$ \\
Additive scale & & $(0.146-0.479)$ \\
RERl & 1.053 & $(1.157-2.801)$ \\
AP & 0.313 & \\
S index & 1.8 & $(1.817-2.575)$ \\
Multiplicative scale & & \\
OR & 2.163 & \\
\hline
\end{tabular}

Model 1: adjusted for age and sex

Model 2: adjusted for age, sex, race, education level, family income, current smoking and drinking status, physical activity, BMI, diabetes, family history of stroke, and atrial fibrillation

$\mathrm{AP}$, attributable proportion due to interaction; $\mathrm{BMI}$, body mass index; $\mathrm{Cl}$, confidence interval; $\mathrm{HLP}$, hyperlipidemia; $\mathrm{HTN}$, hypertension; $\mathrm{OR}$, odds ratio; $\mathrm{RERI}$, relative excess risk due to interaction; $\mathrm{S}$ index, synergy index

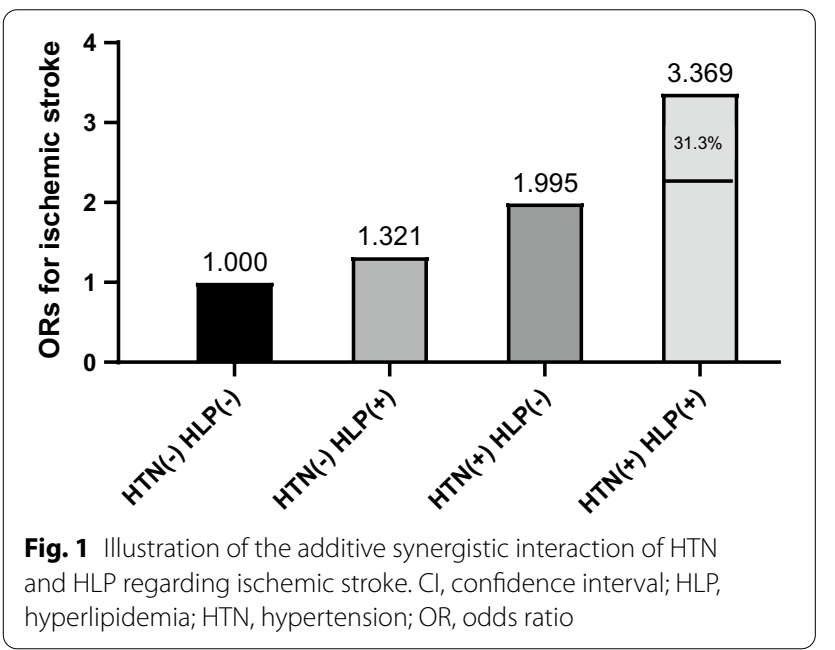

$[34,35]$. Nevertheless, no previous studies examined whether comorbid HTN and HLP are associated with a synergistically increased risk of ischemic stroke in the general population. Our results indicate that in individuals with HLP, HTN is independently associated with ischemic stroke, and vice versa. Most importantly, our findings show that when HTN and HLP coexist, there will be a greater impact on the risk of ischemic stroke compared to the sum of the independent effects.

The underlying mechanism of the synergistic interaction is still unclear, but studies have confirmed that atherosclerosis is the leading cause of increased stroke-related mortality [36]. As a progressive inflammatory disease, atherosclerosis is caused by deposit/plaque accumulation in the intima of blood vessels, which causes arterial narrowing [37]. Its occurrence and development are attributed to various risks including endothelial injury, HLP, and HTN [38]. For example, HLP can lead to the excessive production of reactive oxygen species that damage the endothelium and cause atherosclerosis [39]. Additionally, HTN can prevent vasodilation or increase contraction [40]. Therefore, both HTN and HLP are pathological conditions that cause vascular endothelial damage to persist, impairing vasodilation, increasing the lipid permeability of cells, and causing various vascular diseases oppositely [41, 42]. This situation can eventually lead to artery narrowing, inflammation, and the formation of foam cells and atherosclerosis [43]. Individuals with HTN are also prone to insulin resistance, which inhibits the breakdown of lipids [44]. All of the above factors can explain the pathophysiological relationships involving HTN, HLP, and ischemic stroke, which helps to understand the synergistic interaction between HTN and HLP. This study provides evidence of the synergistic effect of HTN and HLP on the risk of ischemic stroke in the general population.

Our research has several limitations that should be considered when interpreting the results. First, our largescale survey was only conducted in rural areas in China, which may have led to selection bias. Second, in terms of statistical analysis and clinics, we carefully considered the potential confusion in the associations of HTN and HLP with ischemic stroke. Lastly, this was a cross-sectional study, so the results only provide an indication that there is a synergistic effect of HTN and HLP, but the causal relationship still needs verification in prospective longitudinal studies.

\section{Conclusion}

In summary, this study showed that the combined effect of HTN and HLP on ischemic stroke was significantly higher than the sum of their independent effects. The results provide the public with intuitive and straightforward examples to help them to understand the dangerous effects of HTN combined with HLP regarding the risk of 
ischemic stroke. These findings may help individuals to maintain healthy blood pressure and blood lipid levels.

\section{Acknowledgements}

This work was supported by the National Key Research and Development Program of China under Grant 2017 YFC1307600 and the Science and Technology Program of Liaoning Province, China under Grant 2020JH1/10300002. The funding bodies had no role in study design; the collection, analysis, or interpretation of data; writing this manuscript; or the decision to submit this article for publication.

\section{Authors' contributions}

CW analyzed the data and wrote the paper. ZD, NY, CS, SL, DG enrolled participants and collected data. CW were responsible for designing the study and performing data analyses. All authors read and approved the final manuscript.

\section{Availability of data and materials}

The datasets used and/or analyzed during the current study do not contain identifiable data and are available from the corresponding author on reasonable request.

\section{Declarations}

\section{Ethics approval and consent to participate}

The Ethics Committee of China Medical University (Shenyang, China) approved the research protocol. Subjects who had undergone preliminary screening and examination were included after signing a written informed consent form, and all data collection and other procedures complied with ethical standards.

\section{Consent for publication}

Not applicable.

\section{Competing interests}

The authors report no conflicts of interest.

\section{Author details}

'Department of Cardiovascular Medicine, The First Hospital of China Medical University, ShenyangLiaoning 110001, China. ${ }^{2}$ The First Affiliated Hospital, Zhejiang University School of Medicine, Hangzhou 310000, Zhejiang, China.

Received: 22 November 2021 Accepted: 18 January 2022

Published online: 13 February 2022

\section{References}

1. Mathers $C D$, Loncar D. Projections of global mortality and burden of disease from 2002 to 2030. PLoS Med. 2006;3:e442.

2. Liu L, Wang D, Wong KS, Wang Y. Stroke and stroke care in China: huge burden, significant workload, and a national priority. Stroke. 2011;42:3651-4.

3. Wang J, An Z, Li B, et al. Increasing stroke incidence and prevalence of risk factors in a low-income Chinese population. Neurology. 2015;84:374-81.

4. Miller J, Kinni H, Lewandowski C, et al. Management of hypertension in stroke. Ann Emerg Med. 2014;64:248-55.

5. Wright JT Jr, Williamson JD, Whelton PK, et al. A randomized trial of intensive versus standard blood-pressure control. N Engl J Med. 2015;373:2103-16.

6. Zuo Y, Wang A, Wu S, et al. Antihypertensive treatment decrease stroke occurrence: a prospective cohort study. J Hypertens. 2021;39:1652-61.

7. Wei X, Zhang Z, Chong MKC, et al. Evaluation of a package of risk-based pharmaceutical and lifestyle interventions in patients with hypertension and/or diabetes in rural China: a pragmatic cluster randomised controlled trial. PLoS Med. 2021;18:e1003694.

8. Miao J, Zang X, Cui X, et al. Autophagy, hyperlipidemia, and atherosclerosis. Adv Exp Med Biol. 2020;1207:237-64.
9. Lewis A, Segal A. Hyperlipidemia and primary prevention of stroke: does risk factor identification and reduction really work? Curr Atheroscler Rep. 2010;12:225-9.

10. Scandinavian Simvastatin Survival Study Group. Randomised trial of cholesterol lowering in 4444 patients with coronary heart disease: the Scandinavian Simvastatin Survival Study (4S). Lancet. 1994;344:1383-9.

11. Asplund K, Karvanen J, Giampaoli S, et al. Relative risks for stroke by age, sex, and population based on follow-up of 18 European populations in the MORGAM Project. Stroke. 2009;40:2319-26.

12. Goldstein LB. Statins and ischemic stroke severity: cytoprotection. Curr Atheroscler Rep. 2009;11:296-300.

13. Rosvall M, Persson M, Östling G, et al. Risk factors for the progression of carotid intima-media thickness over a 16-year follow-up period: the Malmö Diet and Cancer Study. Atherosclerosis. 2015;239:615-21.

14. Wada S, Koga M, Toyoda K, et al. Factors associated with intima-media complex thickness of the common carotid artery in Japanese noncardioembolic stroke patients with hyperlipidemia: the J-STARS Echo Study. J Atheroscler Thromb. 2018;25:359-73.

15. Meschia JF, Brott T. Ischaemic stroke. Eur J Neurol. 2018;25:35-40.

16. Guzik A, Bushnell C. Stroke Epidemiology and risk factor management. Continuum (Minneap Minn). 2017;23:15-39.

17. Wang C, Du Z, Ye N, et al. Using the atherogenic index of plasma to estimate the prevalence of ischemic stroke within a general population in a rural area of China. BioMed Res Int. 2020;2020:7197054.

18. Li Z, Bai Y, Guo X, et al. Alcohol consumption and cardiovascular diseases in rural China. Int J Cardiol. 2016;215:257-62.

19. Yu S, Guo X, Li GX, et al. Lower or higher HDL-C levels are associated with cardiovascular events in the general population in rural China. Lipids Health Dis. 2020;19:152.

20. Chen S, Guo X, Zhang X, et al. Association between elevated serum alanine aminotransferase and cardiometabolic risk factors in rural Chinese population: a cross-sectional study. BMC Cardiovasc Disord. 2015;15:65.

21. Zhang N, Chen Y, Guo X, et al. Metabolic abnormalities, but not metabolically healthy obesity, are associated with left ventricular hypertrophy. Heart Lung Circ. 2017;26:251-7.

22. Du Z, Xing L, Lin $M$, et al. Estimate of prevalent ischemic stroke from triglyceride glucose-body mass index in the general population. BMC Cardiovasc Disord. 2020;20:483.

23. Joint Committee for Developing Chinese Guidelines on Prevention and Treatment of Dyslipidemia in Adults. [Chinese guidelines on prevention and treatment of dyslipidemia in adults]. Zhonghua Xin Xue Guan Bing Za Zhi. 2007:35:390-419.

24. Kendler KS, Gardner CO. Interpretation of interactions: guide for the perplexed. Br J Psychiatry. 2010;197:170-1.

25. Knol MJ, van der Tweel I, Grobbee DE, et al. Estimating interaction on an additive scale between continuous determinants in a logistic regression model. Int J Epidemiol. 2007;36:1111-8.

26. Andersson T, Alfredsson L, Källberg H, et al. Calculating measures of biological interaction. Eur J Epidemiol. 2005;20:575-9.

27. Assmann SF, Hosmer DW, Lemeshow S, et al. Confidence intervals for measures of interaction. Epidemiology. 1996;7:286-90.

28. Li R, Chambless L. Test for additive interaction in proportional hazards models. Ann Epidemiol. 2007:17:227-36.

29. Rothman KJ, Greenland S, Walker AM. Concepts of interaction. Am J Epidemiol. 1980;112:467-70.

30. Vandenbroucke JP, von Elm E, Altman DG, et al. Strengthening the reporting of observational studies in epidemiology (STROBE): explanation and elaboration. PLoS Med. 2007;4:e297.

31. M'Barek L, Sakka S, Megdiche F, et al. Traditional risk factors and combined genetic markers of recurrent ischemic stroke in adults. J Thromb Haemost. 2021. https://doi.org/10.1111/jth.15448.

32. Turin TC, Okamura T, Afzal AR, et al. Hypertension and lifetime risk of stroke. J Hypertens. 2016;34:116-22.

33. Zhang R, Zhong C, Zhang Y, et al. Immediate antihypertensive treatment for patients with acute ischemic stroke with or without history of hypertension: a secondary analysis of the CATIS randomized clinical trial. JAMA Netw Open. 2019;2:e198103.

34. Tietge UJ. Hyperlipidemia and cardiovascular disease: inflammation, dyslipidemia, and atherosclerosis. Curr Opin Lipidol. 2014;25:94-5. 
35. Huang $Y Q$, Huang $J Y$, Liu L, et al. Relationship between triglyceride levels and ischaemic stroke in elderly hypertensive patients. Postgrad Med J. 2020;96:128-33.

36. Chow YL, Teh LK, Chyi LH, et al. Lipid metabolism genes in stroke pathogenesis: the atherosclerosis. Curr Pharm Des. 2020;26:4261-71.

37. Safar ME. Arterial aging-hemodynamic changes and therapeutic options. Nat Rev Cardiol. 2010;7:442-9.

38. Atabek ME, Akyürek N, Eklioglu BS, et al. Impaired systolic blood dipping and nocturnal hypertension: an independent predictor of carotid intima-media thickness in type 1 diabetic patients. J Diabetes Complicat. 2014;28:51-5.

39. Huang Q, Qin L, Dai S, et al. AIP1 suppresses atherosclerosis by limiting hyperlipidemia-induced inflammation and vascular endothelial dysfunction. Arterioscler Thromb Vasc Biol. 2013;33:795-804

40. Kassan M, Galán M, Partyka M, et al. Endoplasmic reticulum stress is involved in cardiac damage and vascular endothelial dysfunction in hypertensive mice. Arterioscler Thromb Vasc Biol. 2012;32:1652-61.

41. Yang HY, Bian YF, Zhang HP, et al. LOX-1 is implicated in oxidized lowdensity lipoprotein-induced oxidative stress of macrophages in atherosclerosis. Mol Med Rep. 2015;12:5335-41.

42. Ndisang JF, Wang R. Age-related alterations in soluble guanylyl cyclase and CGMP pathway in spontaneously hypertensive rats. J Hypertens. 2003;21:1117-24

43. Hurtubise J, McLellan K, Durr K, et al. The different facets of dyslipidemia and hypertension in atherosclerosis. Curr Atheroscler Rep. 2016;18:82.

44. Reaven GM. Relationship between insulin resistance and hypertension. Diabetes Care. 1991;14(Suppl 4):33-8.

\section{Publisher's Note}

Springer Nature remains neutral with regard to jurisdictional claims in published maps and institutional affiliations.

- fast, convenient online submission

- thorough peer review by experienced researchers in your field

- rapid publication on acceptance

- support for research data, including large and complex data types

- gold Open Access which fosters wider collaboration and increased citations

- maximum visibility for your research: over $100 \mathrm{M}$ website views per year

At BMC, research is always in progress.

Learn more biomedcentral.com/submissions 